\title{
Word frequency and memory: Effects on absolute versus relative order memory and on item memory versus order memory
}

\author{
NEIL W. MULLIGAN \\ Southern Methodist University, Dallas, Texas
}

\begin{abstract}
Word frequency can produce opposite effects on recognition and order memory: Low-frequency words produce greater recognition accuracy, whereas high-frequency words produce superior order memory. The present experiments further delineate the relationship between word frequency and order memory. Experiment 1 indicates that low-frequency words produce worse performance on a measure of absolute order memory but not on a test of relative order, which is consistent with the idea that different forms of information underlie different types of order judgments (Greene, Thapar, \& Westerman, 1998). Experiment 2 contrasted high-, low-, and very low-frequency words on recognition memory and absolute order memory. In comparison with high-frequency words, low-frequency words enhanced recognition, whereas very low-frequency words did not. Both low- and very low-frequency words, however, produced worse memory for absolute order. Thus, the relationship between frequency and item memory is an inverted U-shaped function, whereas the relationship between frequency and absolute order memory is direct. This implies that the item-enhancing effects of lower word frequency may be dissociated from its order-disrupting effects.
\end{abstract}

A primary reason that memory researchers are interested in word frequency is that it produces opposite effects on recognition and recall. Low-frequency words produce better recognition memory, whereas high-frequency words produce superior recall (at least in pure-list designs, as discussed below) (see, e.g., Shepard, 1967; and see Gregg, 1976, for an early review). These results have informed debates about the relationship between recall and recognition, and they are traditionally interpreted as strong evidence against single-process models of recognition and recall (e.g., Crowder, 1976; Murdock, 1974). Word frequency effects are also important to theoretical models of recognition memory, such as dual-process models (e.g., Chalmers \& Humphreys, 1998; Gardiner \& Java, 1990; Guttentag \& Carroll, 1997; Jacoby \& Dallas, 1981; Mandler, 1980), as well as to attempts to delineate empirical regularities in recognition memory, such as the mirror effect (e.g., Glanzer, Adams, Iverson, \& Kim, 1993; Greene, 1996; Maddox \& Estes, 1997; Wixted, 1992).

A traditional account of the word frequency effect in recall is that high-frequency words lend themselves to more organizationaland interitem associative processing (Gregg, 1976; Schulman, 1976; Zechmeister \& Nyberg, 1982), types of encoding that are especially useful in recall but that have little impact on recognition (e.g., Hunt \& McDaniel, 1993; Kintsch, 1977). Several results are consis-

The author was partially supported by Grant 1-R03-MH61324-01 from NIMH during the conducting of this research. Address correspondence to N. Mulligan, Department of Psychology, Southern Methodist University, Dallas, TX 75275-0442 (e-mail: mulligan @ mail.smu.edu). tent with this view. First, in comparison with low-frequency words, high-frequency words produce more category clustering in recall protocols (e.g., Bousfield \& Cohen, 1955), a standard index of organizational processing at encoding (e.g., Hunt \& Einstein, 1981). Second, meaning-based associations are more easily produced in lists of high-frequency items (e.g., Sumby, 1963; Tulving \& Patkau, 1962), enhancing later recall. Third, the index of interitem associative strengths, which is predictive of recall, is greater with high- than with low-frequency lists (Deese, 1960) (see Gregg, 1976, for additional evidence for the importance of organizational and associative bases of the word frequency effect in recall). Finally, and most important for the present purposes, high-frequency words produce greater order memory, as measured by the order reconstruction task (DeLosh \& McDaniel, 1996). Under the standard assumption that this and other tests of absolute order are based on interitem associations (Greene, Thapar, \& Westerman, 1998; Hunt \& McDaniel, 1993; McDaniel, DeLosh, \& Merritt, 2000; see also Li \& Lewandowsky, 1993), these results imply that high-frequency words lead to more interitem associative encoding than do low-frequency words.

This traditional view is expanded in the order-encoding hypothesis proposed by DeLosh and McDaniel (1996). This hypothesis proposes that word frequency produces an encoding tradeoff between information about the item itself and information about the order in which items occur. According to this view, the unusual nature of low-frequency words focuses processing resources on item characteristics and draws resources away from the encoding of serial order and other forms of interitem relational information. 
Because recall is enhanced by serial-order and relational information (Hunt \& McDaniel, 1993), low-frequency items are at a disadvantage. On the other hand, because recognition tests are heavily reliant on item processing and relatively insensitive to order and relational encoding (Engelkamp \& Zimmer, 1994; Hunt \& McDaniel, 1993; Kintsch, 1977), superior recognition of low-frequency words is expected because of their enhanced item encoding.

The order-encoding hypothesis also accounts for another important aspect of the word frequency effect in recallits reliance on experimental design. High-frequency words lead to better recall than do low-frequency words when these item types are presented in pure lists (e.g., Bousfield \& Cohen, 1955; Deese, 1960; DeLosh \& McDaniel, 1996; Shepard, 1967).When high- and low-frequency words are presented in the same (mixed) study list, the word frequency effect in recall is typically eliminated or even reversed (e.g., DeLosh \& McDaniel, 1996; Gregg, 1976; Gregg, Montgomery, \& Castano, 1980; MacLeod \& Kampe, 1996; May \& Tryk, 1970; cf. Watkins, 1977).

According to the order-encoding hypothesis (DeLosh $\&$ McDaniel, 1996), pure lists of low-frequency words produce list-wide disruption of serial-order information, and to the extent that order information contributes to recall, recall is expected to suffer. For mixed lists, however, the order disruption caused by low-frequency items affects not only those items but temporally adjacent highfrequency items. Because serial-order memory is assumed to be based on interitem processing (e.g., Hunt \& McDaniel, 1993; McDaniel et al., 2000), the disruption due to preferential processing of item characteristics for low-frequency words necessarily degrades the overall quality of order encoding for both low- and high-frequency list members, resulting in no relative advantage for high-frequency words. Consistent with this account, DeLosh and McDaniel (1996) demonstrated that order memory was best for highfrequency pure lists, next best for mixed lists, and worst for low-frequency pure lists. Furthermore, order memory was equivalent for high- and low-frequency words in the mixed lists.

Also consistent with the order-encoding account is the observation that the word frequency effect in recognition is not determined by experimental design; a recognition advantage is found for low-frequency words in both purelist and mixed-list designs (see Gregg, 1976; MacLeod \& Kampe, 1996, for reviews). The order-encoding hypothesis proposes that low-frequency words produce greater item encoding in either design type. Because recognition is primarily sensitive to item encoding, and relatively insensitive to serial-order encoding and relational encoding generally (Engelkamp \& Zimmer, 1994; Hunt \& McDaniel, 1993; Kintsch, 1977), it is predicted that the word frequency effect in recognition is not moderated by experimental design. ${ }^{1}$

The order-encoding hypothesis has been applied to several other variables in addition to word frequency. DeLosh and McDaniel (1996) argued that a number of encoding manipulations contrast an unusual and a more typical encod- ing condition (e.g., word frequency, the generation manipulation, bizarre imagery manipulations). The general version of the hypothesis suggests that the unusual encoding condition (e.g., low-frequency words, the generation condition, bizarre sentences) attracts greater processing of item characteristics at the cost of order and relational encoding. This hypothesis was first developed by Nairne, Reigler, and Serra (1991; Serra \& Nairne, 1993) to account for the effects of generation. These authors found that generation enhanced item memory (as measured by recognition) but disrupted memory for serial order (as measured by the order reconstruction task). Several other manipulations featuring an unusual encoding condition have produced the same double dissociation of item and order memory, and this has been interpreted in terms of the order-encoding hypothesis. These manipulations have involved bizarre versus common sentences (McDaniel, Einstein, DeLosh, May, \& Brady, 1995), enacted versus observed events (Engelkamp \& Dehn, 2000), and words presented with versus without perceptual interference (Mulligan, 1999).

Greene et al. (1998) noted that, under the order-encoding hypothesis, disrupted order memory is causally related to superior item memory and thus is an important topic of inquiry for a theoretical understanding of memory. In addition, the order-encoding hypothesis proposes that the encoding dynamics induced by word frequency are typical of those induced by a number of other encoding manipulations. The present experiments were performed to investigate the relationship between word frequency and order memory in light of this hypothesis and recent research on the related manipulations of generation and perceptual interference.

The generality of the finding of disrupted order memory for low- as opposed to high-frequency words, was examined in Experiment 1 by contrasting the effects of word frequency on the order reconstruction test and the relative recency test. The former test requires memory for absolute order, whereas the latter is a test of relative order. Greene et al. (1998) found that generation disrupted order reconstruction (see also Nairne et al., 1991; Serra \& Nairne, 1993) but not relative recency, indicating that the two methods of measuring order memory can be dissociated. Similarly, the perceptual-interference manipulation reduces order memory on tests of absolute order (order reconstruction and order recall tests) but not on the relative recency test (Mulligan, 1999, 2000b). Word frequency, like generation and perceptual interference, has been proposed to affect order encoding (via the item-order tradeoff). Experiment 1 indicates that, like generation and perceptual interference, the effects of word frequency on absolute order memory do not extend to measures of relative order.

Whether disrupted encoding of absolute order is a byproduct of enhanced item encoding, as suggested by the order-encoding hypothesis, was examined in Experiment 2. Recent research on the perceptual-interference effect (Mulligan, 2000b) and the generation effect (Mulligan, 2000a) suggest that this may not always be the case. In 
these studies, Mulligan implemented versions of the perceptual-interference and generation manipulations that do not produce the typical enhancements to item memory. These versions of the manipulations still disrupted absolute order memory in comparison with appropriate control conditions. Thus, for the generation and perceptualinterference manipulations, the enhancements to item memory were dissociated from the disruption to order memory. Whether or not such a dissociation would obtain for word frequency was investigated in Experiment 2.

\section{EXPERIMENT 1}

In the present study, the methodology of Nairne et al. (1991; DeLosh \& McDaniel, 1996; Engelkamp \& Dehn, 2000; Greene et al., 1998; Mulligan, 1999, 2000b) was adapted to compare the effects of word frequency on memory for absolute and relative order. The experiment consisted of multiple study-test blocks, in which each study list was composed of eight high- or low-frequency words (varied between subjects). After a brief distractor task, a test of absolute order (the order reconstruction test) or relative order (the relative recency test) was presented. In the former test, the study items were presented in a scrambled order and the subject's task was to reconstruct the original studied order of the words. In the latter test, two words from the study list were presented and the subject's task was to determine which was presented later in the list.

\section{Method}

Participants. Sixty-four undergraduates at Southern Methodist University participated in exchange for course credit in psychology courses.

Materials and Design. Word frequency (high vs. low) was varied between subjects, and test type (relative recency vs. order reconstruction) was varied within subjects. The stimulus materials consisted of 208 high-frequency and 208 low-frequency words between 4 and 10 letters in length, chosen from the MRC Psycholinguistic Database (Coltheart, 1981). Kučera-Francis (1967) frequencies were higher than 50 per million for the high-frequency words and between 1 and 8 per million for the low-frequency words. Lowand high-frequency words were relatively low on concreteness and imagery, and matched on these ratings (between 100 and 350 on the MRC Psycholinguistic scales; Coltheart, 1981). Mean concreteness for the high- and low-frequency words were 291 and 280, respectively. The corresponding means for imagery were 306 and 292 . Each set of items was randomly divided into 26 study lists of 8 words each. Within each study list, items were randomly assigned to serial position.

The experiment consisted of 26 study-test blocks. The first two blocks were practice and were excluded from the analysis, leaving 24 critical blocks. Half of the study lists were followed by a relative recency test, and half by an order reconstruction test. The ordering of the tests was random, with the constraint that no more than two consecutive blocks included the same test. During the study portion, the type of test could not be anticipated. Two sets of the high-frequency and low-frequency study-test blocks were created in order to counterbalance study lists across test conditions. Thus, across subjects, each study list appeared equally often in both test conditions.

For the relative recency tests, two test items were chosen from the study list. Following Greene et al. (1998), the two test items were separated by four positions in the study list (i.e., the test pair occupied Study Positions 1 and 5, 2 and 6, 3 and 7, or 4 and 8). This produces moderate performance on the relative recency task, allowing one to avoid ceiling or floor effects. The 12 test pairs were evenly divided over the four study positions across lists.

Procedure. Participants were tested individually. The experiment consisted of a series of study-test blocks, each block composed of a study list, a distractor task, and a memory test. Each study list started with a BEGINNING OF LIST message displayed for $2 \mathrm{sec}$. Eight study trials ensued, in which each word was presented for $3 \mathrm{sec}$ followed by a blank screen for $100 \mathrm{msec}$. After the eighth study trial, an END OF LIST message was displayed for $2 \mathrm{sec}$.

Following the study list, the distractor task was presented. This task consisted of categorizing a series of single digits (randomly chosen from the range 1-9) as odd or even. Each digit was presented at the center of the computer screen for $1,000 \mathrm{msec}$ followed by a blank screen for $100 \mathrm{msec}$. The participants were instructed to indicate whether the digit was odd or even by pressing "o" or "e" on the computer's keyboard. The distractor task continued for 27 trials (lasting $29.7 \mathrm{sec}$ ).

On half the blocks, the distractor task was followed by the relative recency test. The participant was presented with the test pair and was asked to indicate which word had been shown later in the study list. The two words were presented next to each other in the center of the computer screen. On half the trials, the more recent item was on the left; on the other half, on the right. The test pair stayed on the computer screen until the participant responded.

On the other half of the blocks, the distractor task was followed by the order reconstruction test, in which all eight study words were presented simultaneously on the computer screen in a new, random order. The participants were instructed to reorder the words to recreate the original order of presentation in the study list. The participants were provided with a test sheet on which to write their answers. The test sheet consisted of a set of blank spaces, numbered 1 through 8 . The participants were asked to write the words on the sheet, using each word once. The words stayed on the computer screen until the participant completed the test, whereupon the test sheet was removed and the participant pressed the space bar to continue to the next study list.

\section{Results and Discussion}

In order to interpret the relative-recency test, it is important to first consider the results of the order reconstruction test. Accuracy in this test is measured by the proportion of study words allocated to their original position, and is presented in Figure 1 as a function of word frequency and serial position on the study list. The central result is better performance for high-than for low-frequency words, replicating the results of DeLosh and McDaniel (1996). Averaged over serial positions, mean proportions correct were .60 and .45 for the high- and low-frequency words, respectively. The proportions correct were submitted to a $2 \times 8$ analysis of variance (ANOVA), with serial position as a within-subjects factor and word frequency as a between-subjects factor. The analysis revealed significant main effects of word frequency, $[F(1,62)=13.73$, $\left.M S_{\mathrm{e}}=0.2081\right]$ and serial position $[F(7,434)=27.40$, $M S_{\mathrm{e}}=0.0453$ ], the latter an effect typical for this test (e.g., Mulligan, 1999; Nairne et al., 1991). The interaction was not significant $(F=1.25)$.

The mean proportions correct on the relative recency test were .66 and .68 for the high- and low-frequency words, respectively, a nonsignificant difference $(F<1)$. 


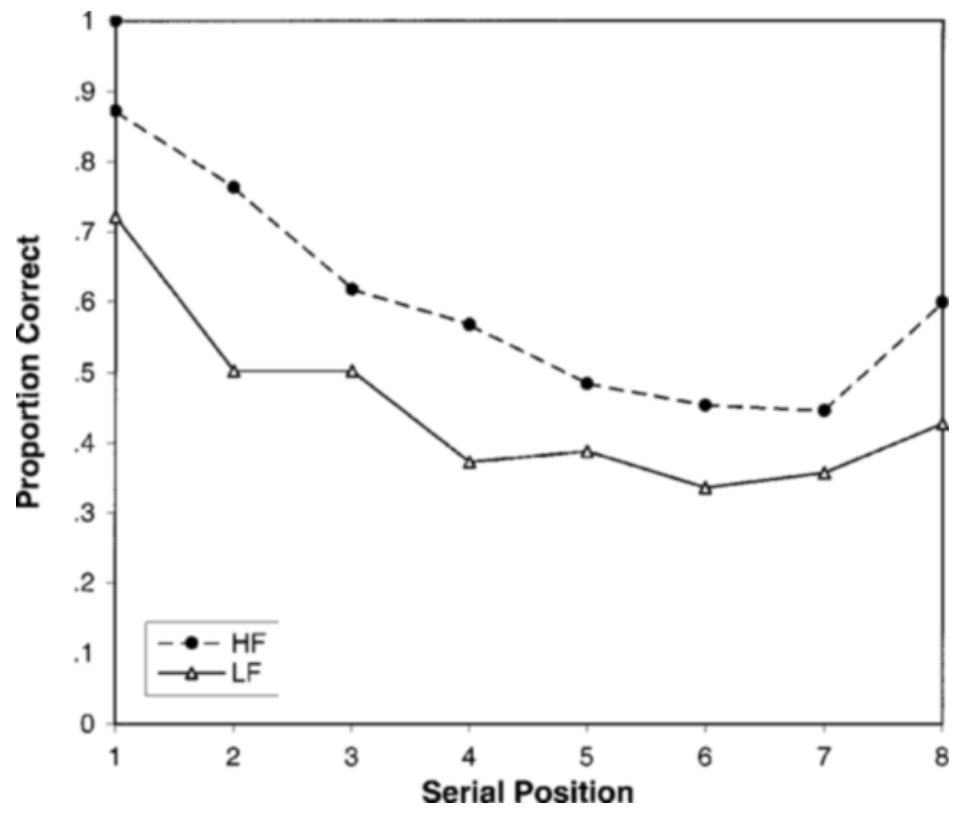

Figure 1. Experiment 1: Mean order reconstruction scores as a function of word frequency and serial position. HF, high frequency; LF, low frequency.

Although not critical in the present design, the effects of serial position are reported here for completeness. For the high-frequency group, mean proportions correct were .59, $.62, .74$, and .70 , for test pairs from Study Positions 1 and 5,2 and 6,3 and 7 , and 4 and 8 , respectively. For the lowfrequency group, the corresponding proportions were .58 , $.69, .71$, and .73 . The effect of serial position was significant, $\left[F(3,186)=2.72, M S_{\mathrm{e}}=0.0972\right]$ indicating increased accuracy for later study positions (Mulligan, 2000b; cf. Greene et al., 1998). The interaction of serial position and word frequency was nonsignificant $(F<1)$.

Before interpreting the apparent dissociation between the order reconstruction and relative recency tests, it is important to first consider the power. For order reconstruction, the effect size of word frequency was $d=.94$. The power to detect an effect of this size for the relative recency effect was $.97(\alpha=.05$, one-tailed, $N=32$ ) (Cohen, $1988)$. In addition, accuracy in the relative recency test was nonsignificantly greater in the low- than in the highfrequency group, a direction opposite that found with the order reconstruction test. This argues that the divergent results of the order reconstruction and relative recency tests were not simply due to insufficient power. In turn, it might be argued that the relative recency test is an insensitive measure of order memory. However, two points militate against this view. First, the test is sensitive enough to register the effects of serial position (the present experiment; Mulligan, 2000b). In addition, Greene (1996) found that words led to significantly greater performance than did nonwords on this task. Most relevant for the present purposes is Greene's (1996, Experiment 2) difference between pure lists of words versus pure lists of nonwords, which yielded an effect size of $d=1.94$. The power of the present relative recency test to detect an effect of this size exceeds .99. The power to detect an effect just half that size is .98 .

The central result of Experiment 1 was that frequency produced a dissociation between two measures of order memory, affecting a measure of absolute order but leaving unaffected a measure of relative order. These results are consistent with those reported for generation and perceptual interference, two other manipulations hypothesized to produce item-order encoding tradeoffs. Both of these manipulations reduce performance on the order reconstruction test (Mulligan, 1999; Nairne et al., 1991; Serra \& Nairne, 1993) and on the order recall test (Mulligan, 2000b), but not on the test of relative recency (Greene et al., 1998; Mulligan, 2000b). ${ }^{2}$

\section{EXPERIMENT 2}

The order-encoding hypothesis implies that disrupted order encoding is a by-product of enhanced item encoding. However, recent research on the perceptual-interference and generation manipulations has indicated that this might not be the case, and that disrupted order encoding may be dissociated from enhanced item encoding. This is illustrated by the perceptual-interference effect. The standard perceptual-interference encoding condition consists of presenting a study word on a computer screen for a very brief time (e.g., $100 \mathrm{msec}$ ) followed by a backward mask (e.g., a row of Xs). This condition is contrasted with an intact condition, in which the study item is presented for a longer duration (e.g., $2.5 \mathrm{sec}$ ) without backward mask- 
ing. In both cases, the subject's task is to identify the word. On later tests of recognition or recall, the perceptualinterference condition produces better memory than does the intact condition; this is the typical perceptual-interference effect (e.g., Mulligan, 1996; Nairne, 1988). However, if the backward mask is delayed (so that it no longer interferes with perception; e.g., there is a word-mask SOA of $266 \mathrm{msec}$ ), the perceptual-interference effect is eliminated in recall (Hirshman, Trembath, \& Mulligan, 1994) and recognition (Mulligan, 2000b). Thus, delaying the mask produces a version of the perceptual interference manipulation that does not enhance item memory. However, in comparison with the intact condition, this encoding condition disrupts order memory to the same extent as does the standard perceptual interference manipulation (Mulligan, 2000b). Similar results are obtained with generation. Mulligan (2000a) found a generation effect in recognition for words but not for nonwords (a typical finding; see, e.g., McElroy \& Slamecka, 1982; Nairne, Pusen, \& Widner, 1985). However, in comparison with reading, generating words and nonwords led to the same-sized deficit on order reconstruction (Mulligan, 2000a).

In the case of word frequency, it is known that lowfrequency words, relative to high-frequency words, enhance item memory and disrupt order memory (at least for absolute order) (e.g., DeLosh \& McDaniel, 1996); this is the item-order tradeoff envisioned by the order-encoding hypothesis. The present experiment was designed to determine whether very low-frequency words, which do not enhance item encoding, would disrupt encoding of absolute order. Over a wide range of word frequency, the relationship between frequency and accuracy in recognition memory is an inverted U-shaped function; low-frequency words produce the highest recognition accuracy, and highand very low-frequency words produce lower accuracy (e.g., Chalmers, Humphreys, \& Dennis, 1997; Mandler, Goodman, \& Wilkes-Gibbs, 1982; Rao \& Proctor, 1984; Schulman, 1976; Wixted, 1992). Thus, very low-frequency words do not typically enhance item memory relative to high-frequency items. Experiment 2 was designed to examine whether very low-frequency words would persist in disrupting order memory and thus demonstrate the dissociation of enhanced item memory and disrupted order memory found with the perceptual-interference and generation manipulations.

The design of Experiment 2 was similar to that of Experiment 1 , in which the study lists consisted of high-, low-, or very low-frequency words. Following $30 \mathrm{sec}$ of distraction, the participant was presented with either a test of absolute order memory (the order reconstruction test) or a row of asterisks indicating no test on the current trial. The untested lists were included in an end-of-session recognition test (a measure of item memory).

\section{Method}

Participants. Seventy-two undergraduates at Southern Methodist University participated in exchange for course credit in psychology courses.
Materials and Design. Word frequency (high, low, and very low) was varied between subjects, and test type (order reconstruction vs. recognition) was varied within subjects. The critical stimulus words consisted of 96 high-frequency, 96 low-frequency, and 96 very low-frequency words, all between 4 and 10 letters in length. The high- and low-frequency words were a subset of the critical items used in Experiment 1, with frequencies higher than 50 per million for the high-frequency words and between 1 and 8 per million for the low-frequency words. Recall that these items were low in imagery and concreteness. This is important, because concrete words produce better performance on the order reconstruction test than abstract words do (Neath, 1997). Because very low-frequency (and unfamiliar) words are presumably of low concreteness and imagery, high- and low-frequency words of low concreteness and imagery provide the best comparison groups. The high- and low-frequency words were matched on concreteness and imagery, with mean concreteness ratings of 278 and 286, and mean imagery ratings of 304 and 297 for the high- and low-frequency words, respectively. The very low-frequency words were chosen as in earlier studies (e.g., Mandler et al., 1982; Rao \& Proctor, 1984). These were English words from the Shorter Oxford English Dictionary (with a status of "rare," according to the MRC Psycholinguistic Database; Coltheart, 1981) that do not appear in the Kučera and Francis (1967) norms. Only items that the experimenter believed would be unfamiliar to participants were used (cf. Mandler et al., 1982; Rao \& Proctor, 1984).

For each frequency condition, the 96 critical items were randomly divided into three sets of 32 . Two of the sets were used to produce the study lists, one to be tested with the order reconstruction test and the other to be tested in the end-of-session recognition test. The third set was used to provide new items on the recognition test. Three versions of the materials were created by cycling each set through each testing condition (i.e., order reconstruction, old on recognition, new on recognition). The two study sets were randomly divided into four study lists, yielding a total of eight study lists with eight words each.

The first part of the experiment consisted of 10 study-test blocks, 8 critical blocks preceded by 2 practice blocks. Practice words were selected by the same criteria as those for the critical items. Each study list was followed by either an order reconstruction test or a line of asterisks (signifying no test in this block; these study items were tested in the end-of-session recognition test). The blocks were randomly ordered, with the constraint that no more than two consecutive study blocks could appear in the same test condition. The second part of the experiment was the end-of-session recognition test, consisting of the 32 studied items from the untested blocks, randomly intermixed with 32 new items of the same frequency (high, low, or very low).

Procedure. The study portion and distractor task of each study-test block were the same as in Experiment 1. On half the blocks, the distractor task was followed by the order reconstruction test, which was also identical to that in Experiment 1. On the other half of the blocks, the distractor task was followed by a line of asterisks. The asterisks remained on the computer screen for $5 \mathrm{sec}$, after which a message appeared, prompting the participant to hit the space bar to continue. On these blocks, the participants had been instructed simply to wait for the next study list. During presentation of the

Table 1

Experiment 2: Performance on Recognition Memory Test as a Function of Word Frequency

\begin{tabular}{lccc}
\hline $\begin{array}{c}\text { Word } \\
\text { Frequency }\end{array}$ & Hits & False \\
Alarms & $d^{\prime}$ \\
\hline High & .63 & .25 & 1.07 \\
Low & .70 & .16 & 1.60 \\
Very low & .57 & .24 & 0.92 \\
\hline
\end{tabular}


study items, the participants did not know whether the list would be followed by order reconstruction or asterisks. After the last studytest block, the participants received the recognition test. They were told that they would be tested for their memory of the words that they had read in the earlier part of the experiment. The participants were told that they would see a series of words on the computer screen. They were asked to indicate which had been presented earlier by typing the "y" key for old words and the "n" key for new words. Each test word was presented individually, centered on the computer screen, and remained until the participant responded.

\section{Results and Discussion}

Before interpreting the results of the order reconstruction task, it is important first to consider the results of the recognition test, particularly because the study conditions of the present paradigm differ from the traditional listlearning paradigm. The recognition results are presented in Table 1 . An analysis of $d^{\prime}$ recognition accuracy revealed a significant effect of word frequency $[F(2,71)=18.43$, $M S_{\mathrm{e}}=0.1652$ ]. Post hoc contrasts (using Fisher's least significant difference) indicated that accuracy was significantly greater in the low-frequency condition than in either the high-or the very low-frequency condition, and that the latter two conditions did not significantly differ. The same pattern of results was obtained with corrected hits (hits - false alarms) as the measure of accuracy. Thus the present study replicates prior research with respect to item recognition; recognition accuracy was an inverted Ushaped function of word frequency, with highest accuracy for words of relatively low frequency and lower accuracy for words of high and very low frequency (e.g., Chalmers et al., 1997; Mandler et al., 1982; Rao \& Proctor, 1984;
Schulman, 1976; Wixted, 1992; Zechmeister, Curt, \& Sebastian, 1978). ${ }^{3}$

Figure 2 presents the results of the order reconstruction test. In contrast to the inverted U-shaped function between recognition accuracy and frequency, accuracy on order reconstruction is a direct function of frequency, with higher levels of frequency associated with greater accuracy. A 2 (word frequency) $\times 8$ (serial position) ANOVA revealed a main effect of serial position $\left[F(7,483)=29.76, M S_{\mathrm{e}}=\right.$ 0.0439 ] as in Experiment 1, and, more importantly, a main effect of word frequency $\left[F(1,69)=10.90, M S_{\mathrm{e}}=0.2238\right]$ (the interaction was not significant, $F=1.08$ ). Post hoc contrasts indicated that accuracy at each level of frequency significantly differed from that for the others with means of $.58, .46$, and .36 , for the high-, low-, and very low-frequency words, respectively.

Contrasting the results of the high- and low-frequency conditions produces a picture concordant with the orderencoding view. Specifically, low-frequency words led to greater item memory (as measured by recognition) but worse order memory (at least as measured by order reconstruction; see Experiment 1). This aspect of the results is consistent with a processing tradeoff in which greater item encoding comes at the cost of less order encoding. However, the story is complicated by consideration of the very low-frequency condition. Here, a condition that led to worse item memory (than that obtained with lowfrequency words) also produced worse order memory (than that for either high- or low-frequency words). Consequently, this aspect of the results resists interpretation in terms of a simple processing tradeoff. The present results

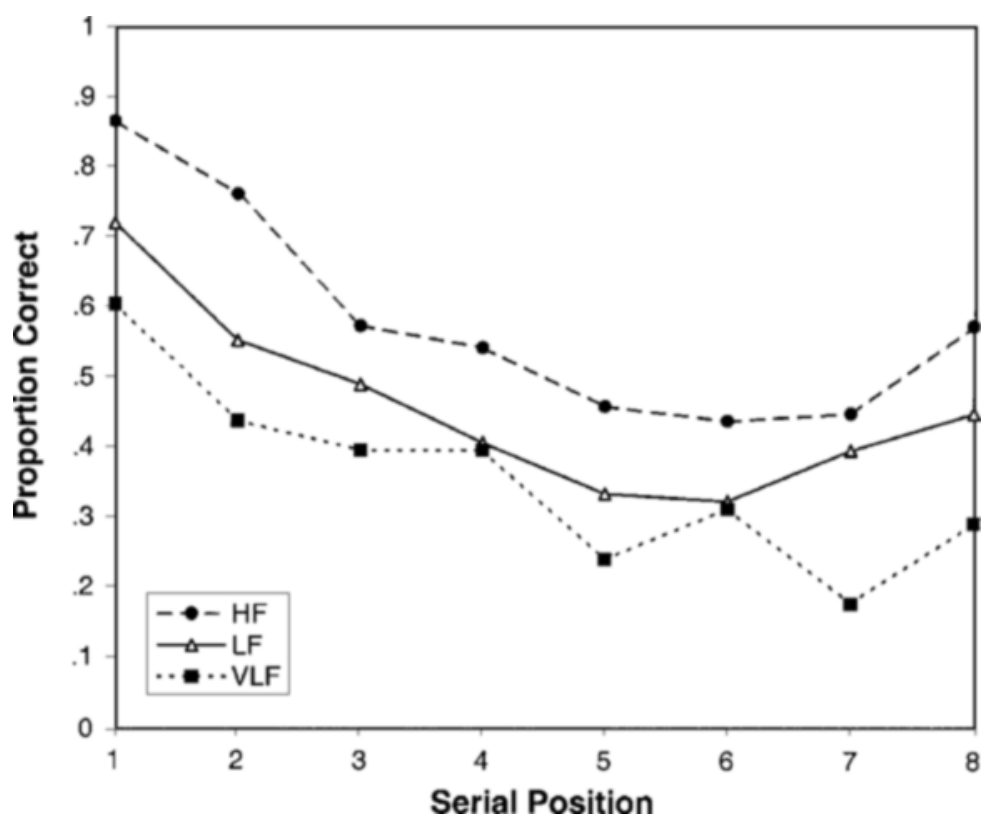

Figure 2. Experiment 2: Mean order reconstruction scores as a function of word frequency and serial position. HF, high frequency; LF, low frequency; VLF, very low frequency. 
represent a dissociation of the positive (item) and negative (order) effects of word frequency.

\section{GENERAL DISCUSSION}

Word frequency is among the encoding manipulations found to have opposite effects on item and order memory (others include generation, perceptual interference, bizarre imagery, self-enactment) (see, e.g., Burns, 1996; DeLosh \& McDaniel, 1996; Engelkamp \& Dehn, 2000; McDaniel et al., 1995; Mulligan, 1999; Nairne et al., 1991; Serra \& Nairne, 1993). The order-encoding hypothesis has been proposed as a unifying account of the similar effects of these varied manipulations (e.g., DeLosh \& McDaniel, 1996; Engelkamp \& Dehn, 2000; see discussion in McDaniel et al., 2000). In the present two experiments, the effects of word frequency on order memory were examined in light of this hypothesis, and the results place important constraints on its application to word frequency.

The results of Experiment 1 question the generality of the order deficit produced by low frequency. Although low-frequency words led to worse performance than did high-frequency words on the order reconstruction test, low- and high-frequency words led to equivalent performance on the relative recency test. The present dissociation of measures of absolute and relative order joins others that have been produced by variables encompassed by the order-encoding hypothesis. Greene et al. (1998) found that generation disrupted performance on order reconstruction but not on a test of relative recency. Mulligan (2000b) found that the perceptual interference manipulation produced the same result. The present results are consistent with the view that there are distinct aspects of encoding supporting different forms of order memory (see, e.g., Greene et al., 1998; Li \& Lewandowsky, 1993, 1995). It has been argued that tests of absolute order (such as order reconstruction and order recall) are performed by serial recall of the list (Greene et al., 1998). Such serial recall relies heavily on interitem associations (e.g., Lewandowsky \& Murdock, 1989; Li \& Lewandowsky, 1993). As discussed in the introduction, it has long been noted that lower word frequency disrupts the formation of interitem associations, reflected in such findings as reduced category clustering (see Gregg, 1976, for a review). Consequently, it would be expected that lower frequency words would lead to reduced recall of absolute position.

The processes underlying recall of relative order may not involve the retrieval of all items in the list or rely on interitem associations (as in serial recall) (Greene et al., 1998; Li \& Lewandowsky, 1993), although the relevant processes are less well understood. Greene et al. suggest that only the to-be-compared items are retrieved and that the judgment is made on the basis of item-position associations, contextual information, or, perhaps, total information retrieved. The present results suggest that low frequency does not disrupt these judgments (although the inclusion of nonwords probably would; see Greene, 1996).
Research on source memory for incidental features of stimuli (such as whether a word has been presented visually or aurally) indicate that low-frequency words are not impaired in the encoding of contextual information, and that they may in fact lead to enhanced contextual encoding (Guttentag \& Carroll, 1997).

Experiment 2 was motivated by a different aspect of the order-encoding hypothesis. This hypothesis proposes that item and order information are involved in a tradeoff produced by a shared encoding mechanism (e.g., Burns, 1996; DeLosh \& McDaniel, 1996; Engelkamp \& Dehn, 2000; Nairne et al., 1991; Serra \& Nairne, 1993). In this view, the aspects of an encoding condition that induce enhanced item processing are assumed to be the same as those that disrupt order encoding. The results of Experiment 2 appear contrary to this view. Specifically, in comparison with high-frequency words, low-frequency words led to better item memory and worse (absolute) order memory, whereas very low-frequency words produced worse memory for both item and order information. Thus, the effects of frequency on item and order memory cannot be described well in terms of a simple tradeoff. Rather, the effect of frequency on item memory can be dissociated from the effect of frequency on order memory. This result coheres with results of studies (Mulligan, 2000a, 2000b) in which versions of the generation and perceptual identification manipulations that did not enhance item memory persisted in disrupting memory for order (as measured by the order reconstruction test).

The idea of a processing tradeoff could be supplemented with consideration of the preexisting representation of the study items, although even this augmented version of the order-encoding account does not comport with all the extant data. Specifically, the order reconstruction results may be accommodated by appealing to the traditional view that the ease of forming interitem associations is a direct function of frequency (see, e.g., Gregg, 1976; Schulman, 1976). To the extent that such associations underlie memory for absolute order (see above), the observed decrease in order memory with decreasing word frequency is expected. In the case of item recognition, it has been argued that stimuli must possess preexisting, meaningful representations to produce enhanced item encoding and distinctiveness (e.g., Chalmers \& Humphreys, 1998; Gardiner, Gregg, \& Hampton, 1988; McElroy \& Slamecka, 1982; Nairne et al., 1985; Schulman, 1976). Thus, it could be the case that low- and even very low-frequency words induce an encoding tradeoff between item and order information, but that for unfamiliar (and essentially meaningless) very low-frequency words, the item representations are not available or are insufficient to render the stimulus distinctive. Under this view, the dissociation obtained in Experiment 2 implies different representational bases for item and order memory but not necessarily different encoding mechanisms or processes.

Although this account preserves the basic features of the order-encoding view, it does not have the generality 
claimed by the original hypothesis (DeLosh \& McDaniel, 1996; Engelkamp \& Dehn, 2000). The original orderencoding view was designed to unify a common pattern of enhanced item memory and disrupted order memory produced by an array of encoding manipulations (DeLosh \& McDaniel, 1996; McDaniel et al., 2000). The present extension of the order-encoding view, however, does not apply to the results of the perceptual-interference manipulation (Mulligan, 2000b). Similar to the results of the present experiments, the enhanced item memory and disrupted order memory produced by perceptual interference can be dissociated. As reviewed earlier, delaying the mask in the perceptual-interference condition eliminates the effect in item memory but produces as large a disruption of order memory as does the standard perceptual-interference condition (Mulligan, 2000b). Thus, the lack of enhanced item memory cannot be attributed to the use of materials without preexisting representations; the same words that produced enhanced item memory in the standard perceptualinterference condition failed to do so in the delayed-mask condition. Consequently, the results of the perceptualinterference manipulation resist interpretation in terms of an encoding tradeoff within a common encoding mechanism, and thus undermine the generality of the augmented order-encoding view. Additional research is required in order to determine whether a general version of the orderencoding hypothesis can be developed in light of the present line of research (Mulligan, 2000a, 2000b), or whether special-case explanations are required for each of the encoding manipulations that produce item-order tradeoffs.

\section{REFERENCES}

Bousfield, W. A., \& Cohen, B. H. (1955). The occurrence of clustering in the recall of randomly arranged words of different frequenciesof-usage. Journal of General Psychology, 52, 83-95.

Burns, D. J. (1996). The item-order distinction and the generation effect: The importance of order information in long-term memory. American Journal of Psychology, 109, 567-580.

Chalmers, K. A., \& Humphreys, M. S. (1998). Role of generalized and episode specific memories in the word frequency effect in recognition. Journal of Experimental Psychology: Learning, Memory, \& Cognition, 24, 610-632.

Chalmers, K. A., Humphreys, M. S., \& Dennis, S. (1997). A naturalistic study of the word frequency effect in episodic recognition. Memory \& Cognition, 25, 780-784.

CoHEN, J. (1988). Statistical power analysis for the behavioral sciences. Hillsdale, NJ: Erlbaum.

Coltheart, M. (1981). The MRC PsycholinguisticDatabase. Quarterly Journal of Experimental Psychology, 33A, 497-505.

Crowder, R. G. (1976). Principles of learning and memory. Hillsdale, NJ: Erlbaum.

DeEse, J. (1960). Frequency of usage and number of words in free recall: The role of association. Psychological Reports, 7, 337-344.

DeLosh, E. L., \& McDAniel, M. A. (1996). The role of order information in free recall: Application to the word-frequency effect. Journal of Experimental Psychology: Learning, Memory, \& Cognition, 22, 1136-1146.

EngelKamp, J., \& Dehn, D. A. (2000). Item and order information in subject-performed tasks and experimenter-performed tasks. Journal of Experimental Psychology: Learning, Memory, \& Cognition, 26, 671-682.

ENGElKAMP, J., \& ZimmeR, H. D. (1994). The human memory: A multimodal approach. Seattle: Hogrefe \& Huber.

Gardiner, J. M., Gregg, V. H., \& Hampton, J. A. (1988). Word fre- quency and generation effects. Journal of Experimental Psychology: Learning, Memory, \& Cognition, 14, 687-693.

GARDINER, J. M., \& JAVA, R. I. (1990). Recollective experience in word and nonword recognition. Memory \& Cognition, 18, 23-30.

Glanzer, M., Adams, J. K., Iverson, G. J., \& Kim, K. (1993). The regularities of recognition memory. Psychological Review, 100, 546-567.

Greene, R. L. (1996). Mirror effect in order and associative information: Role of response strategies. Journal of Experimental Psychology: Learning, Memory, \& Cognition, 23, 687-695.

Greene, R. L., Thapar, A., \& Westerman, D. L. (1998). Effects of generation on memory for order. Journal of Memory \& Language, $\mathbf{3 8}$, 255-264.

GrEGG, V. (1976). Word frequency, recognition and recall. In J. Brown (Ed.), Recall and recognition (pp. 183-216). New York: Wiley.

Gregg, V. H., Montgomery, D. C., \& Castano, D. (1980). Recall of common and uncommon words from pure and mixed lists. Journal of Verbal Learning \& Verbal Behavior, 19, 240-245.

Guttentag, R. E., \& Carroll, D. (1997). Recollection-based recognition: Word frequency effects. Journal of Memory \& Language, 37, 502-516.

Hirshman, E., Trembath, D., \& Mulligan, N. W. (1994). Theoretical implications of the mnemonic benefits of perceptual interference. Journal of Experimental Psychology: Learning, Memory, \& Cognition, 20, 608-620.

Hunt, R. R, \& Einstein, G. O. (1981). Relational and item-specific information in memory. Journal of Verbal Learning \&Verbal Behavior, 19, 497-514.

Hunt, R. R., \& McDaniel, M. A. (1993). The enigma of organization and distinctiveness. Journal of Memory \& Language, 32, 421-445.

JACовY, L. L., \& DALlas, M. (1981). On the relationship between autobiographical memory and perceptual learning. Journal of Experimental Psychology: General, 110, 306-340.

KINTSCH, W. (1977). Memory and cognition. New York: Wiley.

KuČERA, H. \& FrANCIS, W. N. (1967). Computationalanalysis of presentday American English. Providence, RI: Brown University Press.

Lewandowsky, S., \& MuRdock, B. B. (1989). Memory for serial order. Psychological Review, 96, 25-57.

LI, S. C., \& LEWANDOWSKY, S. (1993). Intralist distractors and recall direction: Constraints on models of memory for serial order. Journal of Experimental Psychology: Learning, Memory, \& Cognition, 19, 895-908.

LI, S. C., \& LEWANDOWSKy, S. (1995). Forward and backward recall: Different retrieval processes. Journal of Experimental Psychology: Learning, Memory, \& Cognition, 21, 837-847.

MacLeod, C. M., \& KAMPE, K. E. (1996). Word frequency effects on recall, recognition, and word fragment completion tests. Journal of Experimental Psychology: Learning, Memory, \& Cognition, 22, 132-142.

Maddox, W. T., \& EsTes, W. K. (1997). Direct and indirect effects of stimulus frequency effects in recognition. Journal of Experimental Psychology: Learning, Memory, \& Cognition, 23, 539-559.

Mandler, G. (1980). Recognizing: The judgment of previous occurrence. Psychological Review, 87, 252-271.

Mandler, G., Goodman, G. O., \& Wilkes-GibBs, D. L. (1982). The wordfrequency paradox in recognition. Memory \& Cognition, 10, 33-42.

MAY, R. B., \& TRYK, H. E. (1970). Word sequence, word frequency, and free recall. Canadian Journal of Psychology, 24, 299-304.

McDaniel, M. A., DeLosh, E. L., \& Merritt, P. S. (2000). Order information and retrieval distinctiveness: Recall of common versus bizarre material. Journal of Experimental Psychology: Learning, Memory, \& Cognition, 26, 1045-1056.

McDaniel, M. A., Einstein, G. O., Delosh, E. L., May, C. P., \& BRADY, P. (1995). The bizarreness effect: It's not surprising, it's complex. Journal of Experimental Psychology: Learning, Memory, \& Cognition, 21, 422-435.

McElroy,L. A., \& Slamecka, N. J. (1982). Memorial consequences of generating nonwords: Implications for semantic-memory interpretations of the generation effect. Journal of Verbal Learning \& Verbal Behavior, 21, 243-259.

Mulligan, N. W. (1996). The effects of perceptual interference at encoding on implicit memory, explicit memory, and memory for source. Journal of Experimental Psychology: Learning, Memory, \& Cognition, 22, 1067-1087. 
Mulligan, N. W. (1999). The effects of perceptual interference at encoding on organization and order: Investigating the roles of item-specific and relational information. Journal of Experimental Psychology: Learning, Memory, \& Cognition, 25, 54-69.

Mulligan, N. W. (2000a). The generation effect: Dissociating enhanced item memory and disrupted order memory. Manuscript submitted for publication.

Mulligan, N. W. (2000b). Perceptual interference and memory for order. Journal of Memory \& Language, 43, 680-697.

Murdock, B. B. (1974). Human memory: Theory and data. New York: Wiley.

NAIRNE, J. S. (1988). The mnemonic value of perceptual identification. Journal of Experimental Psychology: Learning, Memory, \& Cognition, 14, 244-255.

NAirne, J. S., Pusen, C., \& Widner, R. L., JR. (1985). Representation in the mental lexicon: Implications for theories of the generation effect. Memory \& Cognition, 13, 183-191.

Nairne, J. S., Reigler, G. L., \& Serra, M. (1991). Dissociative effects of generation on item and order retention. Journal of Experimental Psychology: Learning, Memory, \& Cognition, 17, 702-709.

NeAth, I. (1997). Modality, concreteness, and set-size effects in a free reconstruction of order task. Memory \& Cognition, 25, 256-263.

Rao, K. V., \& Proctor, R. W. (1984). Study-phase processing and the word frequency effect in recognition memory. Journal of Experimental Psychology: Learning, Memory, \& Cognition, 10, 386-394.

Schulman, A. I. (1976). Memory for rare words previously rated for familiarity. Journal of Experimental Psychology: Human Learning and Memory, 2, 301-307.

Serra, M., \& NaIrNe, J. S. (1993). Design controversies and the generation effect: Support for an item-order hypothesis. Memory \& Cognition, 21, 34-40.

SHEPARD, R. N. (1967). Recognition memory for words, sentences, and pictures. Journal of Verbal Learning \& Verbal Behavior, 6, 156-163.

Sumby, W. H. (1963). Word frequency and serial position effects. Journal of Verbal Learning \& Verbal Behavior, 1, 443-450.

Tulving, E., \& PatKau, J. E. (1962). Concurrent effects of contextual constraint and word frequency on immediate recall and learning of verbal material. Canadian Journal of Psychology, 16, 83-95.

Wat Kins, M. J. (1977). The intricacy of memory span. Memory \& Cognition, 5, 529-534.

Wixted, J. T. (1992). Subjective memorability and the mirror effect. Journal of Experimental Psychology: Learning, Memory, \& Cognition, 18, 681-690.

Zechmeister, E. B., Curt, C., \& Sebastian, J. A. (1978). Errors in a recognition memory task are a U-shaped function of word frequency. Bulletin of the Psychonomic Society, 11, 371-373.

Zechmeister, E. B., \& Nyberg, S. E. (1982). Human memory. Monterey, CA: Brooks/Cole.

\section{NOTES}

1. Aspects of the order-encoding hypothesis are similar to other accounts of word frequency effects, such as the attention/likelihood model
(Glanzer et al., 1993) and the notion of indirect effects of frequency on encoding (Maddox \& Estes, 1997). These accounts emphasize that frequency plays a role in the allocation of attention at encoding. Unlike the order-encoding hypothesis, these models were designed to provide a detailed account of the effects of frequency on recognition, especially the mirror effect, rather than a more general account of frequency effects in recall and recognition. Despite the differences in scope, the models are consistent in viewing low frequency as a signal to the encoding system to differentially process item characteristics, with the possible side effect (made explicit in the order-encoding hypothesis) of disrupted processing of order information.

2. Although Greene et al. (1998) argued that the operative difference between the order reconstruction test and relative recency test is that the former but not the latter requires recall of exact study position, another difference is that the order reconstruction test re-presents all eight study items, whereas the relative recency test re-presents only two of the study items. Mulligan (2000b) found that this difference was unimportant in producing dissociations between the two tests. Mulligan (2000b) used an order recall test in which two test items were presented (as in the relative recency test), but in which subjects were required to recall the exact study position of each (rather than produce a relative recency judgment). Perceptual interference disrupted performance on the order recall test (Mulligan, 2000b) as well as on the order reconstruction test (Mulligan, 1999), both of which required recall of exact position information. In contrast, perceptual interference produced no effect on the relative recency judgment. Thus, the operative difference between the order reconstruction and relative recency tests appears to consist in the requirement to recall exact versus relative order information rather than the number of test items presented.

3. Although recognition accuracy is the critical measure for the present purposes, analyses of hits and false alarms may also be provided for completeness. Word frequency exerted a significant effect on both hits $\left[F(2,71)=8.55, M S_{\mathrm{e}}=0.0122\right]$ and false alarms $[F(2,71)=6.07$, $\left.M S_{\mathrm{e}}=0.0080\right]$. Post hoc tests (using Fisher's least significant difference) on hits showed that low-frequency items produced significantly more hits than did either high- or very low-frequency items, and that the latter two conditions did not significantly differ. In contrast, low-frequency items produced significantly fewer false alarms than did either high- or very low-frequency items. Again, the latter two conditions did not significantly differ. In comparison with the results of studies using yes-no recognition and reporting hits and false alarms, the present results are similar to Rao and Proctor's (1984) finding of fewer hits and more false alarms for very low-frequency items than for low-frequency items (the traditional mirror effect; see, e.g., Glanzer et al., 1993). In contrast, Mandler et al. (1982) found fewer hits and fewer false alarms for very low-frequency words. Wixted (1992) speculated that this difference might be due to the use of short versus long retention intervals (immediate testing in Rao \& Proctor, 1984, and the present experiment; 24 or $48 \mathrm{~h}$ in Mandler et al., 1982).

(Manuscript received December 19, 2000; revision accepted for publication July 27, 2001.) 